\title{
SOLAR WIND AND SATURNIAN MOONS SIGNATURES IN THE LONG-PERIODIC MODULATIONS OF SKR
}

\author{
M. L. Khodachenko*, M. Panchenko*, K. G. Kislyakova ${ }^{\dagger}$, \\ A. G. Kislyakov ${ }^{\dagger}$, \\ H. O. Rucker*, and U. Taubenschuss ${ }^{\ddagger}$
}

\begin{abstract}
Long-periodic (LP) modulations of intensity of Saturnian Kilometric Radiation (SKR) recorded by RPWS instrument onboard Cassini spacecraft were studied by means of a combined data analysis algorithm based on a "sliding window" Fourier (SWF) procedure and the nonlinear Wigner-Ville (WV) method. The analyzed SKR data record covers the years 2004-2005. It has been found that SKR has well pronounced impulsive modulation, with quasi-periodic pulses appeared approximately each 10.74 hours and having duration of about 5 hours. The period of pulses, in spite of the overall stability, has sometimes the disturbances within the interval of $10.74-10.76$ hours. By this, the whole impulsive modulation of SKR appeared in 2004-2005 in a form of quasi-regularly repeated modulation activity storms. Time interval between the adjacent modulation activity storms most often was within 8-13 days. Altogether, 55 impulsive modulation activity storms were registered during 2004-2005. Besides of the storms of $10.7 \mathrm{~h}$ pulses, the intensity of SKR has several more long periodic modulations. In particular, $\sim 6-7$ days, $\sim 8-9$ days, $\sim 12-13$ days and $\sim 25-27$ days components were detected. These are connected probably with the quasi-regular character of the storms of $10.74 \mathrm{~h}$ pulses. While the origin of 10.74 hour pulses can be associated with the rotation of Saturn, the long periodic modulation features are very likely connected with the detected similar variations of the solar wind parameters. The last in their turn may be caused by the varying solar surface magnetic activity and rotating streams of high speed solar wind. Besides of that, the performed analysis shows that some Saturnian moons may also have influence on the intensity of SKR. In particular, specific modulations with periods corresponding to orbital motions of close moons (Enceladus, Tethys) as well as Titan, Hyperion and Dione were detected.
\end{abstract}

\footnotetext{
* Space Research Institute, Austrian Academy of Sciences, Schmiedlstrasse 6, A-8042 Graz, Austria

† Lobachevsky State University of Nizhni Novgorod, 23 Prospekt Gagarina, 603950, Nizhni Novgorod, Russia

‡Department of Physics and Astronomy, University of Iowa, Iowa City, IA 52242, USA
} 
